\title{
Disminución del nivel de consciencia secundaria a síndrome de Percheron
}

\author{
Decreased level of consciousness secondary to Percheron syndrome
}

Lourdes Ruiz-Escribano-Menchén ${ }^{1 *}$, José M. Flores-Barragán ${ }^{1}$, Beatriz La Rosa-Salas ${ }^{2}$ y

M. Pilar De Frías-Castellanos ${ }^{3}$

${ }^{1}$ Servicio de Neurología; ${ }^{2}$ Servicio de Medicina Interna; ${ }^{3}$ Servicio de Urgencias. Hospital General Universitario de Ciudad Real, Ciudad Real, España

\section{Introducción}

La alteración del nivel de consciencia constituye un importante motivo de atención en urgencias. Las principales causas son afectación cerebral estructural, disfunción neuronal difusa o causa psicógena ${ }^{1}$. La estructura anatómica más importante en el mantenimiento del nivel de consciencia es el sistema reticular ascendente, constituido por neuronas con origen en la protuberancia y el mesencéfalo que se proyectan hacia el córtex pasando por el tálamo. La consciencia puede alterarse si se afecta algún componente del sistema reticular ascendente 0 sus conexiones ${ }^{2}$.

Presentamos y revisamos el caso de una mujer de 73 años con clínica de disminución de la consciencia y alteración neurooftalmológica diagnosticada de ictus isquémico talámico paramediano bilateral o síndrome de la arteria de Percheron.

\section{Presentación del caso}

Mujer de 73 años de edad, hipertensa en tratamiento con inhibidores de la enzima convertidora de angiotensina y anticoagulada con acenocumarol por prótesis mecánica mitroaórtica y fibrilación auricular permanente. Es traída a urgencias por el servicio de emergencias con deterioro del nivel de consciencia. Sus familiares informan de mayor somnolencia de horas de evolución, dificultad para realizar algunas actividades instrumentales y quejas en relación con diplopía esa misma tarde; niegan cuadro febril reciente, sintomatología infecciosa o posible abuso medicamentoso. A su llegada, las constantes vitales están en rango de normalidad, se encuentra con tendencia al sueño y presenta anisocoria con midriasis arreactiva izquierda, sin objetivarse otra focalidad. Ante una posible etiología vascular, se realizan tomografía computarizada (TC) y angiografía por TC urgentes, sin hallazgos patológicos (Fig. 1A). En el electrocardiograma se objetiva la fibrilación auricular ya conocida, y en la radiografía torácica no hay hallazgos patológicos. El análisis de orina es normal, incluidos tóxicos, y los parámetros de laboratorio no muestran alteraciones, presentando un International Normalized Ratio (INR) de 3.16, acorde a su indicación de anticoagulación. Tras 2 horas de evolución en urgencias, presenta deterioro del nivel de consciencia con puntuación en la escala de coma de Glasgow de 7 (ocular: 1; lenguaje: 2; motor: 4) y anisocoria con midriasis arreactiva alternante, esta vez del ojo derecho, por lo que se repite la TC craneal, que no muestra cambios respecto a la previa. Se solicita ecocardiograma urgente por parte de cardiología, visualizando

\section{Correspondencia:}

*Lourdes Ruiz-Escribano-Menchén

E-mail: lourdesrem92@gmail.com
Fecha de recepción: 01-12-2021

Fecha de aceptación: 10-12-2021 DOI: 10.24875/REIE.21000230
Disponible en internet: 07-03-2022

Rev Educ Investig Emer. 2022;4(Supl 1):70-73 www.medicinadeemergencias.com bajo la licencia CC BY-NC-ND (http://creativecommons.org/licenses/by-nc-nd/4.0/). 


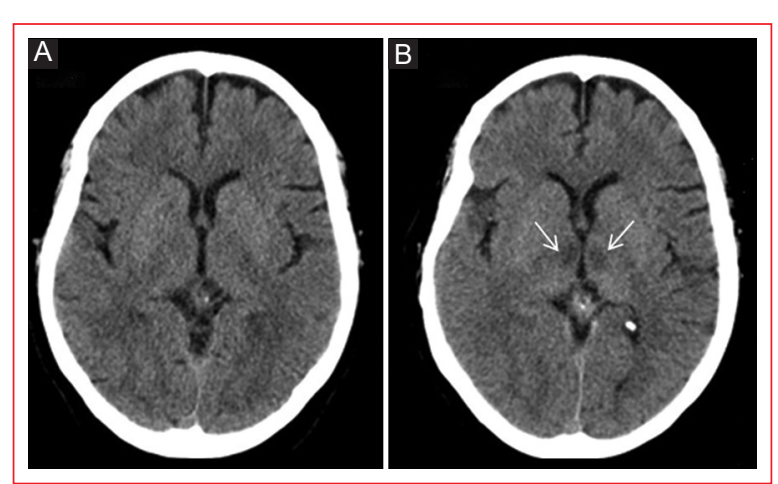

Figura 1. Tomografía computarizada (TC), cortes axiales. A: TC basal urgente sin hallazgos patológicos. B: TC a los 3 días del inicio de los síntomas, en la que se objetivan hipodensidades talámicas paramedianas bilaterales sugestivas de lesiones isquémicas en relación con síndrome de Percheron.

prótesis normofuncionantes y ausencia de verrugas 0 trombos intracavitarios. Las constantes vitales se mantienen estables, sin precisar soporte ventilatorio, ni aparición de pico febril. Posteriormente inicia una mejoría progresiva del nivel de consciencia, aunque presenta amnesia del episodio, confabulación y parálisis de la mirada vertical. Durante su estancia hospitalaria se completa el estudio con un electroencefalograma que no muestra alteraciones, y al estar contraindicada la resonancia magnética por ser portadora de prótesis cardíaca se realiza una TC craneal de control al tercer día, en la que se objetivan hipodensidades talámicas paramedianas bilaterales sugestivas de lesiones isquémicas en relación con síndrome de Percheron (Fig. 1B). Durante el ingreso persisten la parálisis de la mirada vertical y la confabulación, con posterior resolución paulatina. Actualmente se encuentra en seguimiento ambulatorio y muestra deterioro cognitivo leve como secuela.

\section{Discusión}

El tálamo está irrigado por múltiples vasos de pequeño calibre que nacen de la arteria comunicante posterior y de los segmentos P1 y P2 de las arterias cerebrales posteriores. En concreto, el territorio paramediano está irrigado por las arterias talamoperforantes, ramas de P1 (Fig. 2A). Estas arterias tienen una gran variabilidad en cuanto a número y tamaño, pudiendo extenderse a territorios de otras ramas cuando estas no existen. Así mismo, el mesencéfalo rostral está irrigado por las arterias mesencefálicas superiores que nacen de P1 (Fig. 2A) o comparten origen con las arterias talamoperforantes ${ }^{2,3}$ (Fig. 2B). La arteria de Percheron es una variante anatómica descrita en 1973 por Gerard Percheron ${ }^{4}$, consistente en una única arteria talamoperforante que irriga el tálamo medial bilateral y, ocasionalmente, el mesencéfalo rostral (Fig. 2B), produciéndose, en caso de oclusión, un patrón de isquemia característico con afectación talámica paramediana bilateral con o sin afectación mesencefálica.

La prevalencia estimada de la arteria de Percheron es del $4-11.7 \%$ en la población general ${ }^{2}$, y su oclusión es causa del $0.1-2 \%$ de los ictus isquémicos ${ }^{5} \mathrm{y}$ del 22$35 \%$ de los ictus talámicos ${ }^{6}$. No obstante, dada la gran variabilidad clínica secundaria a la compleja irrigación talámica y sus múltiples relaciones con el resto de las estructuras encefálicas, junto con la baja sensibilidad de la TC en la fase aguda, seguramente esté infradiagnosticado, llevando en muchas ocasiones al error diagnóstico (sobre todo en población anciana ante la frecuencia de cuadros confusionales y alteraciones cognitivas).

La alteración del nivel de consciencia (muchas veces fluctuante), los trastornos oculomotores, destacando la parálisis de la mirada vertical (más frecuente, aunque no exclusiva, de la afectación mesencefálica ${ }^{3,7}$ ), y las alteraciones cognitivo-conductuales (principalmente afectación mnésica) constituyen la tríada de presentación más típica.

La afectación de la memoria es frecuente en las lesiones talámicas paramedianas debido a la afectación de distintas estructuras (tracto mamilotalámico y dorsomedial en el territorio paramediano y núcleo anterior en el territorio polar), siendo la amnesia más persistente y profunda cuando hay afectación de todas ellas ${ }^{8}$.

Las alteraciones pupilares son frecuentes en relación con la afectación del núcleo de Edinger-Westphal, los fascículos oculomotores, las fibras simpáticas descendentes o una combinación de estas estructuras ${ }^{9,10}$.

El pronóstico generalmente es bueno, siendo el déficit oculomotor, los trastornos cognitivos y la hipersomnia las secuelas con más frecuencia descritas ${ }^{3,11}$.

El diagnóstico diferencial en el momento agudo incluye causas tóxico-metabólicas y lesiones estructurales que afecten a componentes del sistema reticular ascendente $o$ a sus conexiones. En concreto, el diagnóstico diferencial de las lesiones talámicas bilaterales incluye ictus isquémicos del territorio posterior, trombosis venosas profundas cerebrales, neoplasias infiltrativas 0 lesiones infecto-inflamatorias, las cuales no suelen respetar el territorio vascular típico descrito y muchas de ellas cursan de manera subaguda o crónica ${ }^{3,6}$. 


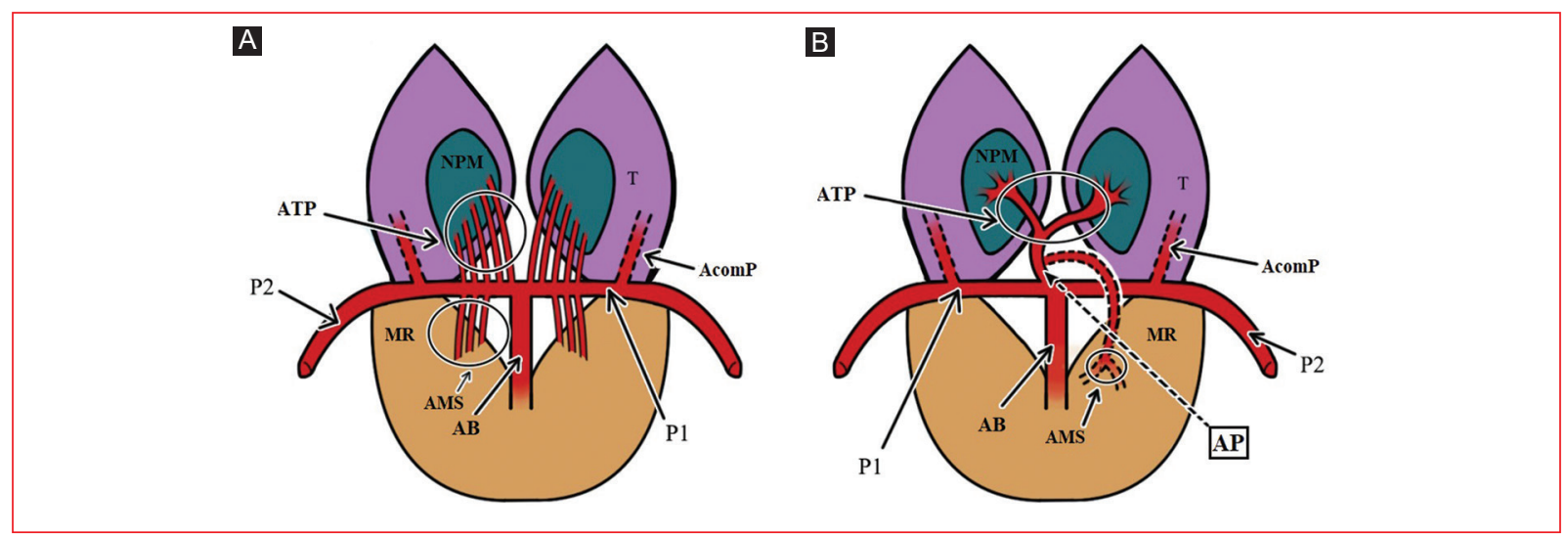

Figura 2. Esquema simplificado del origen de las arterias talamoperforantes y mesencefálicas superiores. A: las arterias talamoperforantes emergen de P1 para irrigar el núcleo paramediano del tálamo y las arterias mesencefálicas superiores emergen de P1 para irrigar el mesencéfalo rostral. B: las arterias talamoperforantes emergen a través de una única rama desde P1 con una posterior ramificación para irrigar los núcleos paramedianos del tálamo, y las arterias mesencefálicas superiores emergen del mismo pedículo de P1 para irrigar el mesencéfalo rostral.

AB: arteria basilar; AcomP: arteria comunicante posterior; AMS: arterias mesencefálicas superiores; $A P$ : arteria de Percheron; ATP: arterias talamoperforantes; MR: mesencéfalo rostral; NPM: núcleo paramediano; T: tálamo.

Por ello, ante la sospecha clínica, si hay disponibilidad, es importante la realización temprana de una resonancia magnética con difusión o una TC de perfusión urgente, ya que el diagnóstico precoz es fundamental para la instauración de un tratamiento rápido y adecuado, incluyendo la fibrinólisis intravenosa ${ }^{12}$ (contraindicada en nuestra paciente por su INR de 3.16 debido al tratamiento activo con acenocumarol). En cuanto a la angio-TC, aunque la visualización de la arteria de Percheron es difícil por su pequeño tamaño y variabilidad anatómica (y por ello su ausencia tampoco indica oclusión $\left.{ }^{3,11}\right)$, es importante realizarla en el momento agudo para descartar una oclusión de gran vaso.

\section{Conclusiones}

Destacamos la importancia de conocer este síndrome e incluirlo en el diagnóstico diferencial en todos los pacientes con bajo nivel de consciencia sin lesiones objetivables en la TC simple urgente inicial, especialmente si hay afectación oculomotora concomitante.

\section{Agradecimientos}

Agradecemos la colaboración de Pablo Ruiz-Escribano Martínez en la realización de la ilustración de la figura 2.

\section{Financiamiento}

El presente trabajo no ha recibido ayudas específicas provenientes de agencias del sector público, sector comercial o entidades sin ánimo de lucro.

\section{Conflicto de intereses}

Los autores declaran que no tienen ningún conflicto de intereses.

\section{Responsabilidades éticas}

Protección de personas y animales. Los autores declaran que para esta investigación no se han realizado experimentos en seres humanos ni en animales.

Confidencialidad de los datos. Los autores declaran que han seguido los protocolos de su centro de trabajo sobre la publicación de datos de pacientes.

Derecho a la privacidad y consentimiento informado. Los autores han obtenido el consentimiento informado de los pacientes y/o sujetos referidos en el artículo. Este documento obra en poder del autor de correspondencia.

\section{Bibliografía}

1. Edlow JA, Rabinstein A, Traub SJ, Wijdicks E. Diagnosis of reversible causes of coma. Lancet. 2014;384:2064-76. 
2. Wong ML, Edlow JA. Artery of Percheron stroke. J Emerg Med 2018;55:114-7.

3. Lazzaro NA, Wright B, Castillo M, Fischbein NJ, Glastonbury CM Hildenbrand PG, et al. Artery of Percheron infarction: imaging patterns and clinical spectrum. AJNR Am J Neuroradiol. 2010;31:1283-9.

4. Percheron G. The anatomy of the arterial supply of the human thalamus and its use for the interpretation of the thalamic vascular pathology. Z Neurol. 1973;205:1-13.

5. García-Grimshaw MA, Peschard-Franco M, Gutiérrez-Manjarrez FA. Bilateral thalamic ischemic stroke secondary to occlusion of the artery of Percheron. Cureus. 2018;10:e2676.

6. Rodríguez EG, Lee JA. Bilateral thalamic infarcts due to occlusion of the artery of Percheron and discussion of the differential diagnosis of bilateral thalamic lesions. J Radiol Case Rep. 2013;7:7-14.
7. Clark JM, Albers GW. Vertical gaze palsies from medial thalamic infarctions without midbrain involvement. Stroke. 1995;26:1467-70.

8. Perren F, Clarke S, Bogousslavsky J. The syndrome of combined polar and paramedian thalamic infarction. Arch Neurol. 2005:62:1212-6.

9. Thurtell MJ, Halmagyi GM. Complete ophthalmoplegia, an unusual sign of bilateral paramedian midbrain-thalamic infarction. Stroke. 2008;39:1355-7.

10. Turner J, Richardson T, Kane I, Vundavalli S. Decreased consciousness: bilateral thalamic infarction and its relation to the artery of Percheron. BMJ Case Rep. 2014;2014:bcr2013201848.

11. De la Cruz C, Márquez M, Aguilar R, Romero M, Valdivieso P. Síndrome de la arteria de Percheron: variabilidad clínica y diagnóstico diferencial. Rev Neurol. 2011;53:193-200.

12. Kostanian V, Cramer SC. Artery of Percheron thrombolysis. AJNR Am J Neuroradiol. 2007;28:870-1. 